\title{
Functional Preserving Sublobectomy: A Novel Method for Sublobectomy
}

\author{
Min Zhang ${ }^{1}$ Liang Chen ${ }^{1}$ Qingchen $W^{1}{ }^{1}$ Mingjian Ge ${ }^{1}$ \\ ${ }^{1}$ Department of Cardiothoracic Surgery, The First Affiliated Hospital \\ of Chongqing Medical University, Chongqing, China \\ Thorac Cardiovasc Surg \\ Address for correspondence Mingjian Ge, MD, Department of \\ Cardiothoracic Surgery, The First Affiliated Hospital of Chongqing \\ Medical University, Yuzhong District, Chongqing 400016, China \\ (e-mail: gemingjiancqyy@126.com).
}
Abstract
Keywords
- functional preserving
- wedge
- sublobectomy

We introduce a new method for sublobectomy. It utilizes the easiness and rapidity of wedge resection, and the accuracy and functional preserving of anatomical segmentectomy. It can preserve lung function with less sacrifice of lung parenchyma.

The advantage of sublobectomy (wedge resection, segmentectomy, subsegmentectomy) in the specific treatment of early lung cancer like ground-glass opacities (GGOs) has been considered increasingly in recent years. ${ }^{1}$ Wedge resection is assumed to be more limited than segmentectomy for peripheral lung tumors, but it is difficult to secure an adequate surgical margin, especially when the tumor is deep inside the tissues. ${ }^{2}$ Moreover, wedge resection does not respect the intrapulmonary anatomy, one or more segments may be resected without attention to the bronchovascular anatomy. The anatomical segmentectomy, however, emphasizes anatomical dissection with individual ligation of hilar structures. It does not transgress intersegmental planes, and there is no mass crushing and suturing of lung parenchyma. However, segmentectomy is technically difficult and time consuming.

Here we introduce a novel method for sublobectomy, which we called: functional preserving sublobectomy (FPSL). We can quickly and accurately resect nonvisible and nonpalpable tumors without using any tumor markings.

\section{Technique Description}

A 52-year-old female patient was admitted to our hospital for a lesion in left lower lobe. The HRCT revealed a 12-mm GGO

received

March 7, 2021

accepted after revision

June 1, 2021 located in $S^{6}$ b but very close to $S^{10}$ a ( Fig. 1A). This was a partial-solid GGO, and the consolidation tumor ratio (CTR) was $50 \%$. Compared with her HRCT 1 year ago, the size of GGO grew from 9 to $12 \mathrm{~mm}$ and the consolidation tumor ratio increased from 30 to $50 \%$. The PET-CT was not recommended for patient. Preoperative 3D-CTBA (three-dimensional computed tomography bronchography and angiography) shows that $B^{6} b$ arises from $B^{6}$ and $B^{10} a$ arises from $B^{10}$ (- Fig. 1B). The $A^{6}$ is subdivided into $A^{6} a, A^{6} b$, and $A^{6} c \cdot A^{10} a$ arises from $A^{10}$ (-Fig. 1C) (-Supplementary Fig. S1, available online only). The segments of $S^{6}$ and $S^{10}$ were shown in - Fig. 1D. The main utility incision and observing port was inserted in the fourth and seventh intercostal space in the anterior axillary line. Two assistant incisions were made in the seventh intercostal space in mid-axillary line and ninth intercostal space in posterior axillary line. The lesion is nonvisible and nonpalpable. The multi-subsegmentectomy of $S^{6} b^{+} S^{10}$ a is technically challenging and time consuming, so we decided to perform a FPSL. Dissection started from the oblique fissure. The interlobe artery was identified. The first branch originating from the interlobe artery is $A^{6}$, which is subdivided into $A^{6} a, A^{6} b$, and $A^{6} c . A^{6} b$ is ligated. Then we continue to explore the basal pulmonary artery, and $A^{10}$ is identified. The first branch of $A^{10}$ is $A^{10} a$, which is also ligated. Then we require the (c) 2021. The Author(s).

This is an open access article published by Thieme under the terms of the Creative Commons Attribution-NonDerivative-NonCommercial-License, permitting copying and reproduction so long as the original work is given appropriate credit. Contents may not be used for commercial purposes, or adapted, remixed, transformed or built upon. (https://creativecommons.org/ licenses/by-nc-nd/4.0/) Georg Thieme Verlag KG, Rüdigerstraße 14, 70469 Stuttgart, Germany 


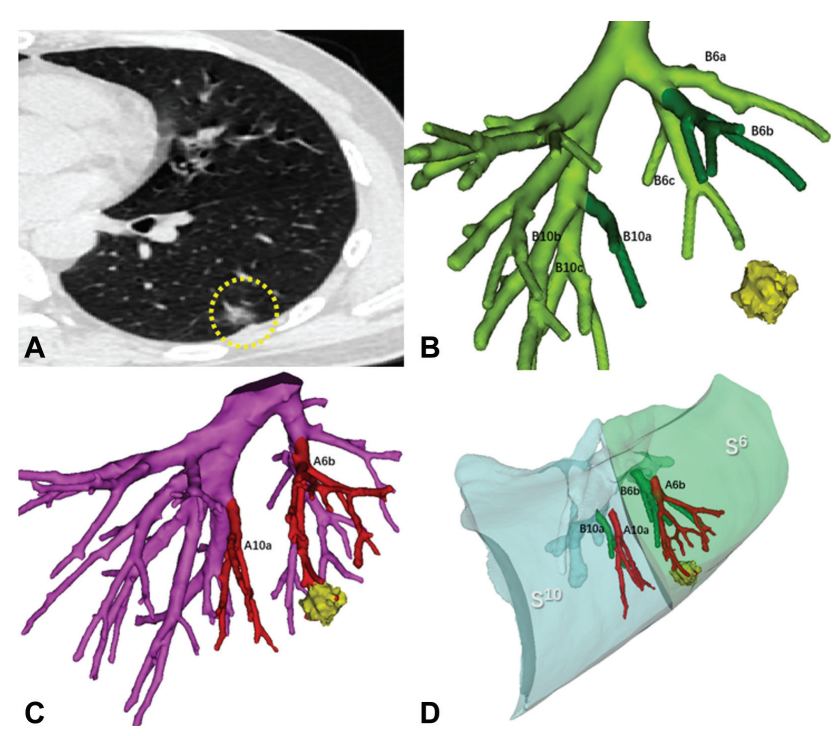

Fig. 1 Computed tomography and 3D-CTBA images (reconstruction with Mimics, Materialise Medical, Leuven, Belgium). (A) A highresolution computed tomography scan revealed a 12-mm GGO between $S^{6} b$ and $S^{10}$ a. (B) $3 D$-CTBA shows that $B^{6} b$ arises from $B^{6}$, and $B^{10}$ a arises from $B^{10}$. (C) The $A^{6}$ is single-stem type, which branches into $A^{6} a, A^{6} b$ and $A^{6} C$. $A^{10} a$ arises from $A^{10}$. (D) The segment of $S^{6+10}$ and the target structures inside. 3D-CTBA, three-dimensional computed tomography bronchography and angiography; GGO, ground-glass opacities. anesthesiologist to ventilate the lung using $100 \%$ oxygen. After 10 minutes, the intersegmental plane is visualized. A wedge resection is performed along the demarcation line. A safe oncological margin is guaranteed (the margin distance is $2.5 \mathrm{~cm}$ ). The final pathological examination shows a result of minimally invasive carcinoma (-Video $\mathbf{1}$ ). The sampling of lymph node (station 12,13) shows a result of no metastasis.

\section{Video 1}

Thoracoscopic right $S^{6} b^{+} S^{10} a$ FPSL resection. FPSL, functional preserving sublobectomy. Online content including video sequences viewable at: https://www. thieme-connect.com/products/ejournals/html/ $10.1055 / \mathrm{s}-0041-1735810$.

\section{Discussion}

From April 2020 to July 2020, 19 patients underwent FPSL in our hospital. In the traditional wedge resection, the border of resection depends subjectively on the surgeon's palpation. By FPSL, we can locate the lesion in a totally different and noninvasive way: the preoperative 3D-CTBA helps us to identify the target vessels penetrating the lesions. By

Table 1 Patients' backgrounds and surgical outcomes

\begin{tabular}{|c|c|c|c|c|c|c|c|c|}
\hline \multirow{2}{*}{$\begin{array}{l}\text { Surgery } \\
\text { Methods (N) }\end{array}$} & \multicolumn{2}{|l|}{$L S^{6} b+S^{10} a$} & \multicolumn{2}{|c|}{$\mathrm{LS}^{1+2} \mathrm{a}+\mathrm{S}^{3} \mathrm{c}$} & \multicolumn{2}{|l|}{$\mathrm{RS}^{9} \mathrm{ai}$} & \multicolumn{2}{|l|}{$\mathrm{RS}^{3} \mathrm{~b}$} \\
\hline & FPSL (7) & AS (6) & FPSL (5) & AS (3) & FPSL (2) & AS (2) & FPSL (5) & AS (4) \\
\hline Age $(y)$ & $56.2 \pm 1.2$ & $48 \pm 2.2$ & $58.5 \pm 4.1$ & $62.8 \pm 4.3$ & $46.1 \pm 3.5$ & $49.8 \pm 4.3$ & $52.8 \pm 4.5$ & $55.1 \pm 2.2$ \\
\hline Size $(\mathrm{mm})$ & 15.3 & 14.8 & 12.3 & 14.7 & 17.3 & 18.7 & 14.3 & 15.9 \\
\hline CTR & $50 \%$ & $45 \%$ & $40 \%$ & $45 \%$ & $45 \%$ & $40 \%$ & $35 \%$ & $40 \%$ \\
\hline Follow-up (month) & 14 & 17 & 29 & 23 & 18 & 23 & 18 & 20 \\
\hline $\begin{array}{l}\text { Thoracoscopic } \\
\text { time (min) }\end{array}$ & $30.1 \pm 2.4$ & $70.8 \pm 2.2$ & $21.3 \pm 3.1$ & $75.4 \pm 4.1$ & $32.3 \pm 0.8$ & $81.2 \pm 4.4$ & $25.3 \pm 3.3$ & $90.5 \pm 5.2$ \\
\hline Blood loss (mL) & $50 \pm 4.6$ & $312 \pm 2.6$ & $45.5 \pm 3.7$ & $251.3 \pm 3.1$ & $55.8 \pm 2.2$ & $350.7 \pm 4.5$ & $31.3 \pm 5.5$ & $303.9 \pm 8.6$ \\
\hline $\begin{array}{l}\text { Conversion to open } \\
\text { surgery }^{a}\end{array}$ & 0 & 1 (LN calcification) & 0 & 1 (LN calcification) & 0 & 0 & 0 & 0 \\
\hline $\begin{array}{l}\text { Median hospital stay } \\
\text { (days) }\end{array}$ & $2.1 \pm 0.4$ & $4.2 \pm 1.1$ & $1.4 \pm 0.5$ & $3.2 \pm 0.8$ & $1.1 \pm 0.2$ & $4.5 \pm 0.8$ & $1.8 \pm 0.7$ & $3.1 \pm 0.8$ \\
\hline Prolonged air leakage ${ }^{\mathrm{b}}$ & 0 & 1 & 0 & 0 & 0 & 0 & 0 & 0 \\
\hline Hemoptysis & 0 & 3 & 0 & 3 & 0 & 3 & 0 & 3 \\
\hline Margin distance $(\mathrm{cm})$ & $3.1 \pm 0.4$ & $3.9 \pm 0.7$ & $2.9 \pm 0.2$ & $3.8 \pm 0.7$ & $3.5 \pm 0.6$ & $3.7 \pm 0.7$ & $4.1 \pm 0.6$ & $3.9 \pm 0.4$ \\
\hline $\operatorname{DBVP}(\mathrm{cm})$ & $2.1 \pm 0.2$ & $5.6 \pm 0.7$ & $1.3 \pm 0.5$ & $4.7 \pm 0.5$ & $1.7 \pm 0.3$ & $4.8 \pm 0.3$ & $1.5 \pm 0.1$ & $3.8 \pm 0.7$ \\
\hline LN sampling number & 2 & 2 & 2 & 3 & 2 & 2 & 3 & 2 \\
\hline Frozen section of LN & \multicolumn{2}{|l|}{ Negative } & \multicolumn{2}{|l|}{ Negative } & \multicolumn{2}{|l|}{ Negative } & \multicolumn{2}{|l|}{ Negative } \\
\hline Pathology & \multicolumn{2}{|c|}{ AIS 6, MIA 7} & \multicolumn{2}{|c|}{ AIS 2, MIA 6} & \multicolumn{2}{|c|}{ AIS 1, MIA 3} & \multicolumn{2}{|c|}{ AIS 3, MIA 6} \\
\hline
\end{tabular}

Abbreviations: AIS, adenocarcinoma in situ; AS, anatomical subsegmentectomy; CTR, consolidation/tumor ratio; DBVP, depth beneath visceral pleura; FPSL, functional preserving sublobectomy; LN, lymph node; MIA, minimally invasive adenocarcinoma.

${ }^{\text {a }}$ The reason of conversion is calcification of the lymph node.

${ }^{\mathrm{b}}$ Prolonged air leakage is air leakage for more than 7 days. 
blocking the target vessels, the circulation of this region is interrupted. The alveoli in which the pulmonary circulation continues can perform gas exchange and absorb oxygen (deflation zone). Alveoli with no pulmonary circulation (target segments) cannot be involved in gas exchange and retain oxygen inside (inflation zone). The border between inflation and deflation area defines the boundary of wedge resection. $^{3,4}$ All patients achieved parenchymal resection margins $\geq 2 \mathrm{~cm}$ or more than or equal to the size of the nodule ( -Table 1). Compared with anatomical segmentectomy, its advantages include less time, less blood loss, and less complications ( $\mathbf{- T a b l e ~} \mathbf{1}$ ). The limitation of this method is that it is only suitable for lesions located near the visceral pleura (-Table $\mathbf{1}$ ).

The FPSL is a balancing strategy between wedge resection and anatomical subsegmentectomy. It utilizes the easiness and rapidity of wedge resection, and the accuracy and precision of anatomical segmentectomy.

\section{Conflict of Interest}

None declared.

\section{References}

1 Khullar OV, Liu Y, Gillespie T, et al. Survival after sublobar resection versus lobectomy for clinical stage Ia lung cancer: an analysis from the National Cancer Data Base. J Thorac Oncol 2015; 10(11):1625-1633

2 Mohiuddin K, Haneuse S, Sofer T, et al. Relationship between margin distance and local recurrence among patients undergoing wedge resection for small $(\leq 2 \mathrm{~cm})$ non-small cell lung cancer. J Thorac Cardiovasc Surg 2014;147(04):1169-1175, discussion $1175-1177$

3 Zhao Y, Xuan Y, Song J, Qiu T, Qin Y, Jiao W. A novel technique for identification of the segments based on pulmonary artery plane combined with oxygen diffusing discrepancy. J Thorac Dis 2019; 11(12):5427-5432

4 Wang C, Cai L, Chen Q et al. No-waiting segmentectomy: an optimized approach for segmentectomy. J Thorac Dis 2021;13 (02):784-788 